\title{
Survey on uric acid in Chinese subjects with essential hypertension (SUCCESS): a nationwide cross-sectional study
}

\author{
Jing Liu ${ }^{1}$, Luyuan Chen ${ }^{2}$, Hong Yuan ${ }^{3}$, Kai Huang ${ }^{4}$, Guangping Li ${ }^{5}$, Ningling Sun ${ }^{6}$, Yong Huo ${ }^{7}$; on behalf \\ of the SUCCESS investigation group
}

${ }^{1}$ Department of Cardiology, Peking University People's Hospital, Beijing, China; ${ }^{2}$ Department of Cardiology, Guangdong Provincial People's Hospital, Guangzhou, China; ${ }^{3}$ Department of Cardiology, The Third Xiangya Hospital of Central South University, Changsha, China; ${ }^{4}$ Department of Cardiology, Union Hospital Affiliated with Tongji Medical College of Huazhong University of Science and Technology, Wuhan, China; ${ }^{5}$ Department of Cardiology, The Second Hospital of Tianjin Medical University, Tianjin, China; ${ }^{6}$ Department of Cardiology, Peking University People's Hospital, Beijing, China; ${ }^{7}$ Department of Cardiology, Peking University First Hospital, Beijing, Chin

Contributions: (I) Conception and design: J Liu, Y Huo; (II) Administrative support: Y Huo; (III) Provision of study materials or patients: All authors; (IV) Collection and assembly of data: All authors; (V) Data analysis and interpretation: J Liu, Y Huo; (VI) Manuscript writing: All authors; (VII) Final approval of manuscript: All authors.

Correspondence to: Prof. Jing Liu. Department of Cardiology, Peking University People’s Hospital, Beijing, China. Email: heartcenter@163.com; Prof. Yong Huo. Department of Cardiology, Peking University First Hospital, Beijing, China. Email: huoyong@263.net.cn.

Background: Hyperuricemia (HUA) is associated with hypertension and increased cardiovascular risk. Current data regarding the prevalence of HUA in Chinese hypertensive patients are lacking. Our study aims to explore the prevalence and determinants of HUA in Chinese hypertensive adults.

Methods: Treatment-naive hypertensive adults or those taking single antihypertensive agent were included in a nationwide cross-sectional study. Basic demographics, antihypertensive medications, serum uric acid (UA), and other parameters were documented.

Results: The overall prevalence rate of HUA was 38.7\% among 33,785 valid cases, 35.1\% for males (UA $>420 \mu \mathrm{mol} / \mathrm{L}$ ), and $45.2 \%$ for females (UA > $360 \mu \mathrm{mol} / \mathrm{L}$ ). A multiple logistic regression analysis, adjusted for demographic and clinical factors (model 1), revealed that female sex [odds ratio (OR), 95\% CI, 1.43, 1.36-1.51], age of $\geq 65$ years $(1.12,1.05-1.19)$, low evaluated glomerular filtration rate [eGFR; 2.06, 1.91-2.23, the lowest [Q1] vs. the highest quartile (Q4)], unmarried (1.58, 1.10-2.27), Western China residency (3.21, 3.33-3.91), longer hypertension duration (1.97, 1.78-2.12, Q4 vs. Q1) and aspirin use (1.21, 1.14-1.29) were associated with HUA. In a multiple logistic regression analysis adjusted for clinical and metabolic parameters (model 2), female sex (OR, 95\% CI, 1.34, 1.27-1.41), age of $\geq 65$ years (1.09, 1.03-1.16), low eGFR (2.35, 2.19-2.52, Q1 vs. Q4), new-onset hypertension (2.01, 1.73-2.33), higher quartile of fasting blood glucose (FBG), triglyceride (TG), low density lipoprotein cholesterol (LDL-C) levels, and body mass index (BMI) were associated with higher risk of HUA $(1.89,1.76-2.03 ; 2.15,1.99-2.31 ; 2.86,2.67-3.06 ; 1.27,1.27-1.36$, respectively, Q4 vs. Q1). Losartan, valsartan, and nifedipine were associated with lower risk of HUA (OR, 95\% CI, 0.77, 0.67-0.88, 0.68, 0.60-0.77; 0.87, 0.77-0.99, 0.79, 0.70-0.89 and 0.80, 0.70-0.91, 0.82, 0.72$0.92)$, respectively, in models 1 and 2 .

Conclusions: The prevalence rate of HUA in Chinese hypertensive patients was 38.7\%. Female sex, aging ( $\geq 65$ years), and low eGFR were independent predictors of HUA. HUA was lower among the patients who were taking losartan, valsartan, and nifedipine. Western region residents, new-onset hypertension, longer hypertension duration, aspirin use, higher FBG, TG, LDL-C levels and BMI were potential risk factors for HUA.

$\wedge$ ORCID: 0000-0002-9714-7708. 
Keywords: Uric acid (UA); hyperuricemia (HUA); hypertension

Submitted Apr 21, 2020. Accepted for publication Oct 16, 2020.

doi: 10.21037/atm-20-3458

View this article at: http://dx.doi.org/10.21037/atm-20-3458

\section{Introduction}

Hypertension is one of the most important risk factors for cardiovascular diseases (CVDs), and is associated with metabolic disorders, including overweight/obesity, abnormal glucose and lipid profiles, as well as elevated blood uric acid (UA) level. Hyperuricemia (HUA) is usually defined as a serum UA level $>420 \mu \mathrm{mol} / \mathrm{L}(7 \mathrm{mg} / \mathrm{dL})$ for males and $>360 \mu \mathrm{mol} / \mathrm{L}(6 \mathrm{mg} / \mathrm{dL})$ for females (1). HUA induces the development of hypertension (2) and is associated with CVD mortality and all-cause mortality (3). Synergistic interactions between hypertension and HUA greatly increase the risks of subclinical atherosclerosis, CVDs, renal insufficiency and all-cause mortality $(2,4,5)$. Therefore, screening and management of HUA in hypertensive patients should be valued.

Few systematic investigations have focused on the prevalence of HUA in the hypertensive population. A recent cross-sectional study in Africa demonstrated that the prevalence of HUA in newly diagnosed, untreated hypertensive patients was $46.9 \%$, which was significantly higher than the $16.9 \%$ in normotensive individuals (6). A cross-sectional survey among a population aged $40-75$ years in Xinyang, China, in 2007 showed that the prevalence rate of HUA in hypertensive patients was $14.1 \%$ in the rural area, and higher in males than in females $(21.5 \%$ and $10.2 \%$, respectively) (7). Big data on the prevalence of HUA in Chinese hypertensive patients are lacking. In addition, previous studies revealed that thiazide diuretics increased the serum UA level (8), while angiotensin II receptor blocker (ARB) losartan decreased the UA level to some degree $(9,10)$, which indicated the contrasting effects of different antihypertensive agents on UA. However, these effects must be evaluated in a larger hypertensive population.

In view of this, the Chinese Cardiovascular Association and Chinese Society of Cardiology initiated the nationwide Survey on Uric aCid in Chinese subjects with ESsential bypertenSion (SUCCESS) study in multiple provinces and cities in June, 2018, aiming to reveal the prevalence and influential factors of HUA in hypertensive patients in the real world and provide reference for the management of
HUA in this population in the future.

We present the following article in accordance with the STROBE reporting checklist (available at http://dx.doi. org/10.21037/atm-20-3458).

\section{Methods}

This was a cross-sectional study using a convenient sampling strategy to select subjects from a hospital-based population. From June to November 2018, hypertensive patients consecutively referred to the outpatient clinics of hospitals in 17 provinces and municipalities (Liaoning, Hebei, Henan, Shandong, Shanxi, Jiangsu, Anhui, Zhejiang, Hubei, Hunan, Fujian, Guangdong, Sichuan, Chongqing, Tianjin, Shanghai, and Beijing) across China were included in the survey. The subjects were required to meet the following criteria: (I) patients aged $\geq 18$ years; (II) blood pressure (BP)-lowering treatment-naive hypertensive patients with $B P$ of $\geq 140 / 90 \mathrm{mmHg}$, measured three times on different days, or those with established essential hypertension who had been taking a single antihypertensive medication regularly for at least 2 weeks; (III) patients who provided written informed consent before participation. Patients with hypertension comorbid with gout or those receiving urate-lowering therapy with xanthine oxidase inhibitors (XOIs), including allopurinol and febuxostat or uricosuric agents such as probenecid or benzbromarone were excluded from the survey.

In the survey, data from the participants were collected by uniformly trained physicians at each clinic, using a questionnaire included the following information: age, sex, educational level, marital status, history of smoking and/ or drinking, history and duration of hypertension, use of antihypertensive agents and aspirin, and so on. Systolic BP, diastolic BP, heart rate, waist circumference (WC), and body mass index (BMI) were measured or calculated at the clinic. Fasting blood glucose (FBG), total cholesterol, triglyceride (TG), high-density lipoprotein cholesterol, low-density lipoprotein cholesterol (LDL-C), blood creatinine, urea nitrogen, UA, and other parameters were obtained through biochemical tests routinely. Estimated glomerular filtration 


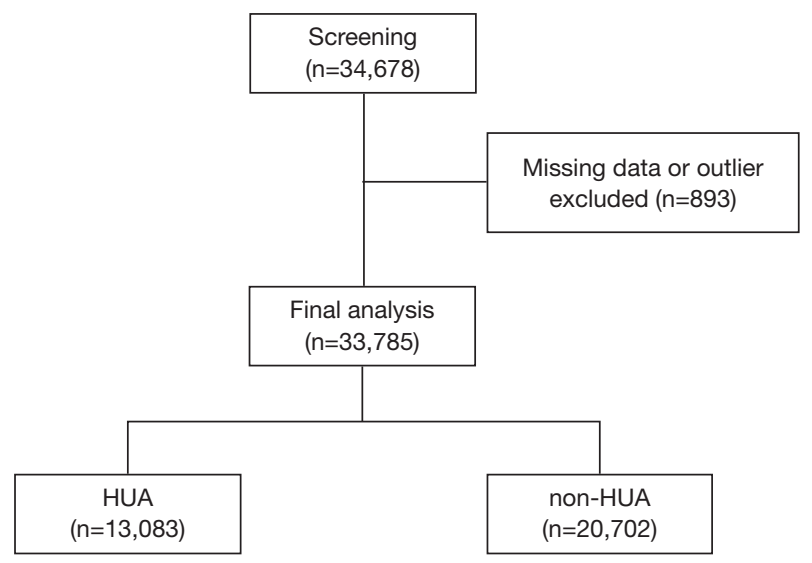

Figure 1 Flowchart of participants.

rate (eGFR) was calculated with the Cockcroft-Gault equation. The participants were divided into the HUA and non-HUA groups, referring to the definition of HUA as a serum UA level of $>420 \mu \mathrm{mol} / \mathrm{L}$ for males or $>360 \mu \mathrm{mol} / \mathrm{L}$ for females. This study was conducted in accordance with the Declaration of Helsinki (as revised in 2013). The study protocol was approved by the central institutional review board of Peking University First Hospital (No. 2018102). Written informed consent was obtained from all the participants.

Statistical analysis: Continuous variables were described as means \pm standard deviation (SD) unless otherwise stated. Categorical variables were described as frequency or percentage. Missing data or outlier values were excluded from the analysis dataset. Chi-square or Fisher's exact tests, $t$ tests, one-way analysis of variance (ANOVA) or nonparametric statistical methods were used for betweengroup parameter comparisons, including population characteristics and serum UA levels. Univariate and multivariate logistic regressions in separate models, adjusted for demographic, clinical and metabolic parameters and other possible confounders, were used to analyze the potential risk or protective factors for HUA. Subgroup population and sensitivity analyses for deferent parameters were also performed. $\mathrm{P}<0.05$ was considered statistically significant. Statistical analyses were conducted using STATA 15.1 (College Station, Texas, USA).

\section{Results}

\section{Baseline characteristics of the patients}

A total of 34,678 patients were enrolled in the survey during the study period. Of them, 893 with missing data or outlier serum UA values (UA $\leq 0$ or $\mathrm{UA}>1,000 \mu \mathrm{mol} / \mathrm{L}$ ) were excluded and 33,785 were included in the statistical analyses (Figure 1). Among the patients included in the analyses, $21,777(64.5 \%)$ were men. More than $90 \%$ of the patients had a history of hypertension and had been receiving a single antihypertensive medication. The median hypertension duration was around 5 years (range, 59-60 months), and the median antihypertensive medication duration was around 3 years (range, 35-38 months). More than $60 \%$ of the patients had high school education or lower, while $16.8 \%$ had an undergraduate degree or higher (Table 1).

\section{Prevalence and gender differences of $\mathrm{HUA}$}

The prevalence rate of HUA was $38.7 \%$ in Chinese hypertensive population, $35.1 \%$ for males and $45.2 \%$ for females, as referring to the definition of HUA with serum UA level of $>420 \mu \mathrm{mol} / \mathrm{L}$ for males or $>360 \mu \mathrm{mol} / \mathrm{L}$ for females. The univariate analysis revealed that the risk of HUA increased by $53 \%$ in the females in comparison with that in the males [odds ratio (OR), 1.53; 95\% CI, 1.46-1.60]. The multivariate logistic regression analysis, with adjustment for demographic and clinical parameters, including age, sex, marital status, educational level, region, eGFR, hypertension duration, antihypertensive medications, history of antihypertensive drug and aspirin use (model 1), revealed that the female hypertensive patients had a significantly higher risk of HUA than the males (OR, 1.43; 95\% CI, 1.36-1.51) (Table 2).

\section{Age and HUA}

With age $<65$ years as control, the univariate logistic regression analysis revealed that age $\geq 65$ years was a risk factor for HUA, with an increased relative risk of $32 \%$ (95\% CI, 1.24-1.39). The multivariate logistic regression analysis revealed that age $\geq 65$ years was still an independent risk factor for HUA even after adjustment for the abovementioned confounders, with a $12 \%$ increased in relative risk (OR, 1.12; 95\% CI, 1.05-1.19).

\section{eGFR and HUA}

When eGFR was categorized into quartiles (indicated by Q1, Q2, Q3 and Q4 from low to high) and the highest quartile $(\mathrm{Q} 4)$ as control, the univariate analysis revealed 
Table 1 Demograhic data and baseline characteristics

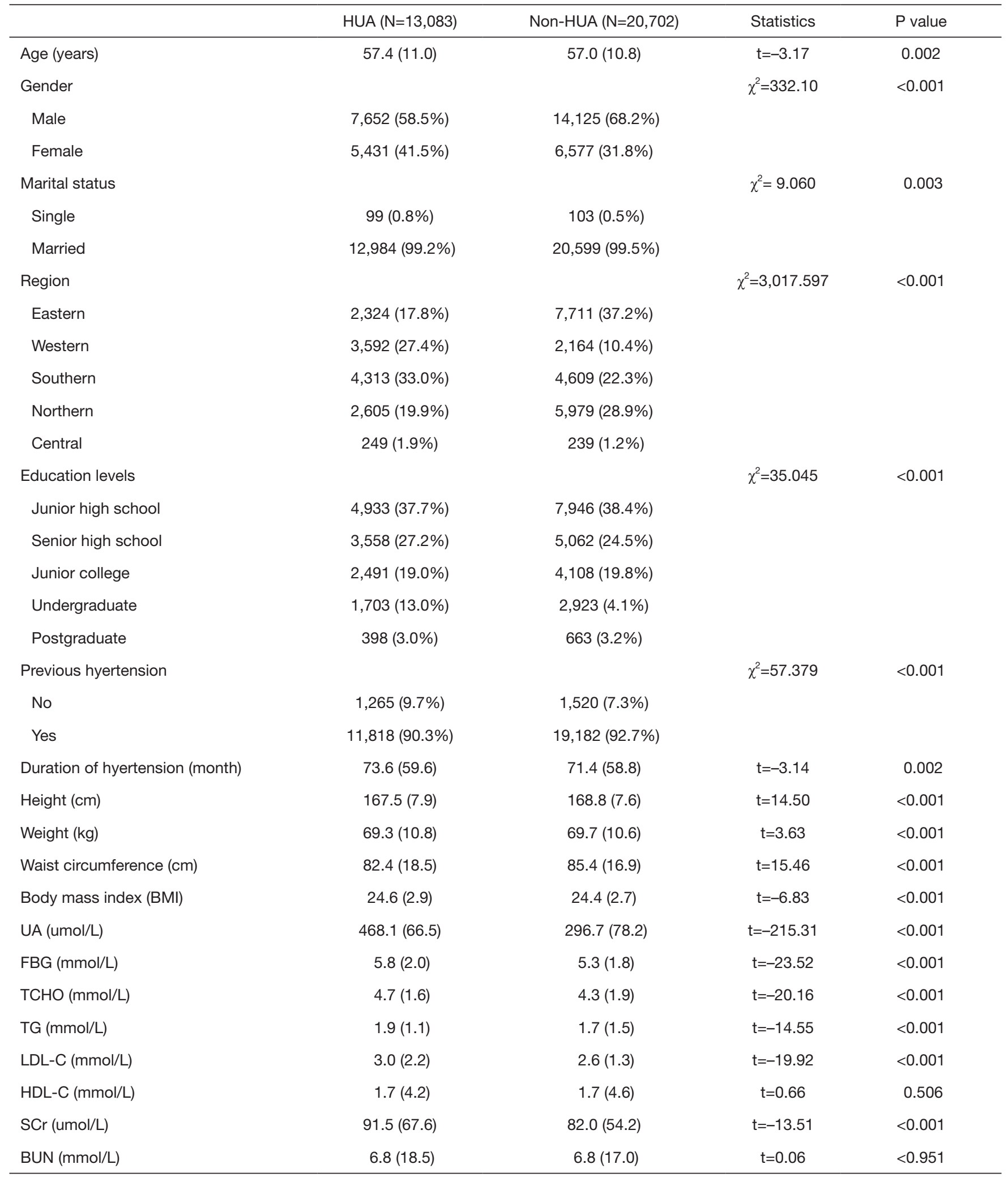

Table 1 (continued) 
Table 1 (continued)

\begin{tabular}{|c|c|c|c|c|}
\hline & HUA $(N=13,083)$ & Non-HUA $(\mathrm{N}=20,702)$ & Statistics & $P$ value \\
\hline AST (IU/L) & $30.6(21.6)$ & $28.3(21.2)$ & $t=-9.37$ & $<0.001$ \\
\hline eGFR (C-G equation) & $88.6(37.2)$ & $101.9(43.9)$ & $t=28.46$ & $<0.001$ \\
\hline Previous use of asirin & & & $\chi^{2}=38.331$ & $<0.001$ \\
\hline Yes & $3,392(25.9 \%)$ & $4,755(23.0 \%)$ & & \\
\hline Antihyertensive agents & & & $\chi^{2}=140.29$ & $<0.001$ \\
\hline ACEls & $1,309(10.0 \%)$ & $1,999(9.7 \%)$ & & \\
\hline ARBs & $4,676(35.8 \%)$ & $8,544(41.3 \%)$ & & \\
\hline Diuretics & $153(1.17 \%)$ & $189(0.91 \%)$ & & \\
\hline others & $134(1.02 \%)$ & $193(0.93 \%)$ & & \\
\hline none & $1,258(9.6 \%)$ & $1,494(7.2 \%)$ & & \\
\hline Duration of treatment (month) & $41.3(34.7)$ & $46.1(37.5)$ & $t=11.12$ & $<0.001$ \\
\hline
\end{tabular}

ACEls, angiotensin converting enzyme inhibitors; ALT, asartate aminotransferase; ARBs, angiotensin recetor blockers; AST, alanine aminotransferase; BMI, body mass index; BUN, blood urea nitrogen; CCBs, calcium channel blockers; C-G equation, Cockcroft-Gault equation; eGFR, estimated glomerular filtration rate; FBG, fasting blood glucose; HDL-C, high density liorotein cholesterol; LDL-C, low density liorotein cholesterol; SCr, serum creatinine; TCHO, total cholesterol; TG, triglyceride; UA, uric acid.

that the ORs of HUA in Q1, Q2 and Q3 were 2.47 (95\% CI, 2.32-2.63), $1.71(1.61-1.83)$ and $1.28(1.20-1.37)$, respectively. The multiple logistic regression analysis revealed that the ORs of HUA in Q1, Q2 and Q3 was $2.06(1.91-2.23), 1.66(1.54-1.79)$ and $1.246(1.16-1.34)$, respectively. The lower the eGFR, the higher the risk of HUA.

\section{Geographical differences in the prevalence of $H U A$}

The prevalence rates of HUA in the five regions across China were $23.2 \%, 62.4 \%, 48.3 \%, 30.3 \%$ and $51.0 \%$ in the Eastern (Shandong, Jiangsu, Anhui, Zhejiang, and Shanghai), Western (Sichuan and Chongqing), Southern (Hunan, Fujian and Guangdong), Northern (Liaoning, Hebei, Shanxi, Tianjin, and Beijing), and Central regions (Henan and Hubei), respectively, lower in the Eastern region but higher in the Western region. With the Eastern region as control, the univariate analysis revealed that the risk of HUA progressively increased in the Northern,
Southern, Central and Western regions, with 4.5-fold increase in the Western region (OR, 5.51; 95\% CI, 5.135.91). The multiple logistic regression analysis revealed that the risk of HUA in the Western region was still the highest (OR, 3.60; 95\% CI, 3.33-3.91).

\section{HUA and educational levels}

We found that the prevalence of HUA was $37.4 \%$ in the hypertensive patients with a college degree or higher (junior college graduates, undergraduates, and postgraduates, 12,286 cases) and $39.5 \%$ in those with a junior high school or high school degree (21,499 cases). With higher educational level (college degree or higher) as control, the univariate analysis revealed an increased risk of HUA in the hypertensive patients with relatively lower educational levels (junior high school or high school; OR, 1.09; 95\% CI, 1.05-1.15). However, the multiple logistic regression analysis did not reveal a correlation between the prevalence of HUA and educational level (OR, 1.02; 95\% CI, 0.96-1.07). 
Table 2 Multivariate logistic regression analysis of the determinants of HUA in hypertensive patients

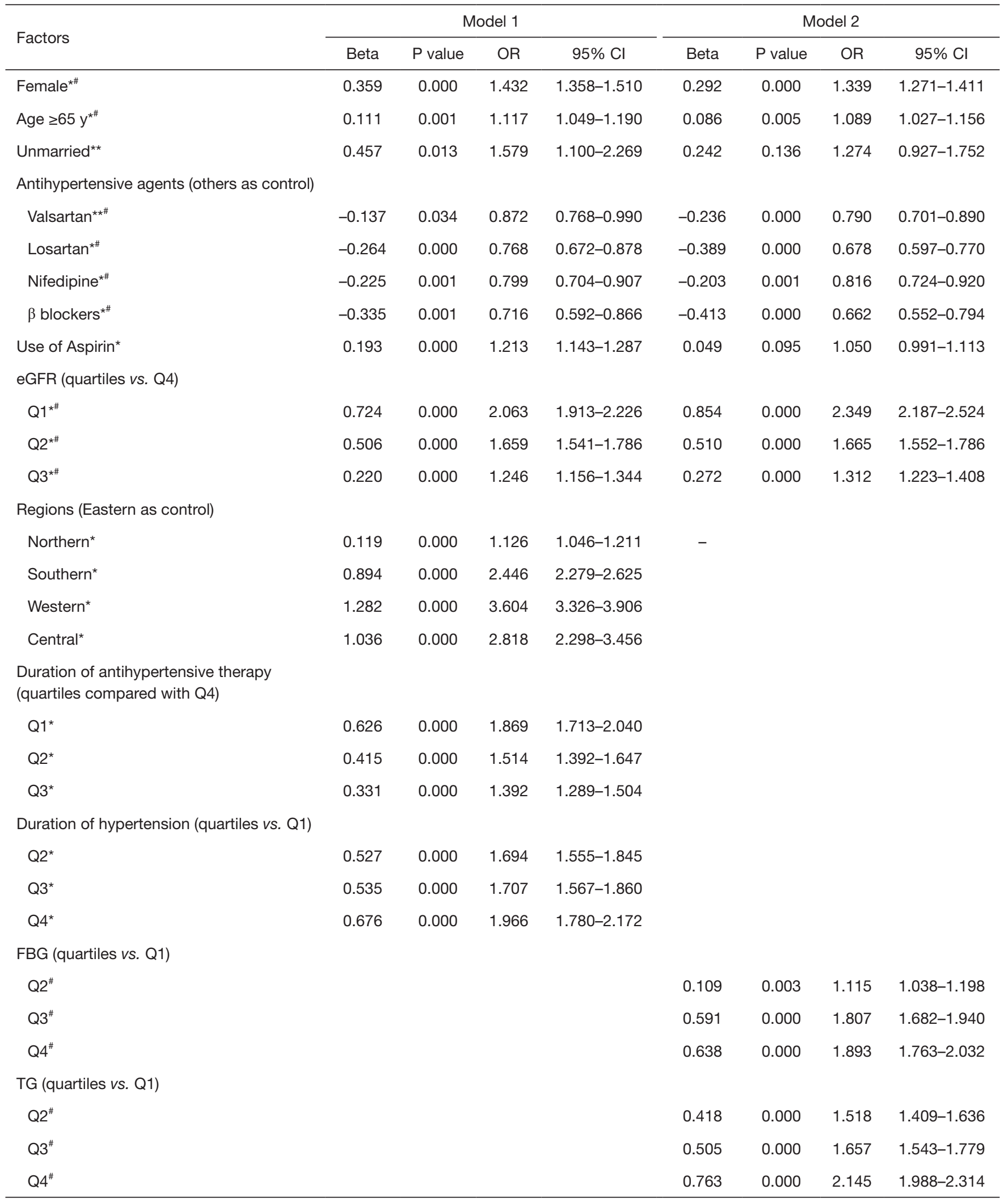

Table 2 (continued) 
Table 2 (continued)

\begin{tabular}{|c|c|c|c|c|c|c|c|c|}
\hline Factors & \multicolumn{4}{|c|}{ Model 1} & \multicolumn{4}{|c|}{ Model 2} \\
\hline \multicolumn{9}{|l|}{ LDL-C (quartiles vs. Q1) } \\
\hline Q2 ${ }^{\#}$ & & & & & 0.365 & 0.000 & 1.440 & $1.341-1.547$ \\
\hline Q3 $^{\#}$ & & & & & 0.583 & 0.000 & 1.791 & $1.665-1.927$ \\
\hline \multicolumn{9}{|l|}{ BMI (quartiles vs. Q1) } \\
\hline Q2 & & & & & 0.055 & 0.117 & 1.057 & $.986-1.133$ \\
\hline Q3 $^{\#}$ & & & & & 0.114 & 0.001 & 1.120 & $1.045-1.201$ \\
\hline $\mathrm{Q}^{\#}$ & & & & & 0.236 & 0.000 & 1.266 & $1.181-1.358$ \\
\hline
\end{tabular}

BMI, body mass index; Cl, confidence interval; eGFR, estimated glomerular filtration rate; FBG, fasting blood glucose; LDL-C, low density lipoprotein cholesterol; OR, odds ratio; TG, triglyceride. *, $\mathrm{P}<0.01$; ${ }^{* *}, \mathrm{P}<0.05$, in model 1, adjustment of age, gender, marital status, education level, region, eGFR, duration of hypertension, history and application of antihypertensive drugs and aspirin; \#, $\mathrm{P}<0.01$, in model 2: adjustment of age, gender, marital status, eGFR, new-onset hypertension, antihypertensive drugs and aspirin application and metabolic parameters.

\section{HUA and bypertension duration}

The survey revealed that the hypertension duration was longer in the HUA group, than in the non-HUA group (mean, 73.6 months, vs. 71.4 months). When hypertension duration was divided into quartiles and with the lowest quartile $(\mathrm{Q} 1)$ as control, the univariate analysis revealed that the ORs of HUA in Q2, Q3 and Q4 were $1.26(95 \%$ CI, 1.18-1.34), 0.98 (0.92-1.05), and 1.14 (1.07-1.22), respectively. The multiple logistic regression analysis revealed that the ORs of HUA in Q2, Q3, and Q4 were 1.69 (95\% CI, 1.56-1.85), 1.71 (1.57-1.86), and 1.97 (1.78-2.12), respectively. The longer the hypertension duration, the higher the risk of HUA.

\section{HUA and antibypertensive medication}

The results showed that BP-lowering treatment-naive hypertensive patients accounted for $8.1 \%$ (2,752 cases) of the total population and those with established hypertension who had been receiving a single antihypertensive drug accounted for $91.7 \%$ (30,996 cases), most of whom had been receiving ARBs $(39.1 \%, 13,220 / 33,785)$, calcium channel blockers (CCBs; 38.2\%, 12,898/33,785) or angiotensin converting enzyme inhibitors (ACEIs; 9.8\%, $3,308 / 33,785)$ and few had been taking $\beta$-blockers $(2.7 \%$,
$901 / 33,785)$, diuretics $(1.0 \%, 342 / 33,785)$ or other antihypertensive drugs $(0.9 \%, 327 / 33,785)$. With BPlowering treatment naivety as control, the univariate analysis revealed that the use of ARBs, ACEIs, CCBs and $\beta$-blockers was associated with decreased risk of HUA in the hypertensive patients. The corresponding ORs were 0.65 (95\% CI, 0.60-0.71), 0.78 (0.70-0.86), 0.81 (0.75-0.88), and 0.61 (0.53-0.72), respectively. The ORs for diuretics and other antihypertensive drugs were 0.96 (95\% CI, 0.77-1.21) and $0.83(0.65-1.04)$, which were not statistically significant. Further analysis revealed that the top four most frequently used antihypertensive agents were valsartan (7,808 cases), nifedipine (controlled-release formulation; 7,341 cases), amlodipine (5,134 cases), and losartan (4,559 cases). The univariate analysis revealed that the use of losartan, valsartan and nifedipine correlated with lower risk of HUA. The corresponding ORs were 0.58 (95\% CI, 0.53-0.64), $0.68(0.62-0.74)$, and $0.73(0.67-0.80)$, respectively. By contrast, amlodipine had no such an effect, with an OR of 0.95 (95\% CI, 0.87-1.05). With other antihypertensive drugs as control, the multivariate logistic regression analysis revealed that only losartan, valsartan, nifedipine and $\beta$-blockers were associated with lower risk of HUA. The ORs were 0.77 (95\% CI, 0.67-0.88), 0.87 (0.77-0.99), 0.80 (0.70-0.91), and $0.72(0.59-0.87)$, respectively. ACEI no 
longer showed a protective effect for HUA, with an OR of 0.97 (95\% CI, 0.85-1.12). Amlodipine and diuretics nonsignificantly increased the risk of HUA, with ORs of 1.09 (95\% CI, 0.96-1.24) and 1.05 (0.81-1.35) respectively. When dummy variables were used in the regression analysis, the multivariate logistic regression analysis indicated that medication with losartan, valsartan and nifedipine, and $\beta$-blockers significantly reduced the risk of HUA, with ORs of 0.68 (95\% CI, 0.60-0.77), 0.79 (0.70-0.89), 0.82 (0.72-0.92), and $0.66(0.55-0.79)$, respectively, $\mathrm{P} \leq 0.001)$, as compared with nonuse of these drugs. ACEI marginally reduced the occurrence of HUA (OR, 0.87; 95\% CI, 0.76-0.99; $\mathrm{P}=0.04)$, whereas amlodipine and diuretics nonsignificantly increased the risk of HUA (OR, 1.06, 95\% CI, 0.93-1.19 and OR, 1.12, 95\% CI, 0.87-1.43, respectively, $\mathrm{P}=0.39)$.

\section{HUA and antibypertensive therapy duration}

When the antihypertensive therapy durations were divided into quartiles, with the highest quartile (Q4) as control, the univariate analysis revealed that the ORs of HUA in Q1, Q2, and Q3 were 0.84 (95\% CI, 0.78-0.89), 1.24 (1.16-1.32), and 0.95 (0.90-1.01), respectively. The multiple logistic regression analysis revealed that short antihypertensive therapy duration was correlated with high risk of HUA [ORs (95\% CI) in Q1, Q2 and Q3 were 1.87 (1.71-2.0), $1.51(1.39-1.65)$ and 1.39 (1.29-1.50), respectively, in contrasted to $\mathrm{Q} 4]$.

\section{HUA and aspirin use}

Of the hypertensive patients who had been taking aspirin regularly $(8,147$ cases $), 41.6 \%$ presented with HUA, whereas of the aspirin-naive patients (25,638 cases), 37.8\% had HUA. With aspirin naivety as control, the univariate analysis revealed that the use of aspirin increased the risk of HUA (OR, 1.19; 95\% CI, 1.13-1.26). The multiple logistic regression analysis revealed that aspirin use was a predictor of HUA, and the relative risk increased by $21 \%$ (OR, 1.21; 95\% CI, 1.14-1.29).

\section{HUA and other metabolic parameters}

The mean $( \pm$ SD) and median serum UA levels were respectively $468.1( \pm 66.5) \mu \mathrm{mol} / \mathrm{L}$ and $456.7 \mu \mathrm{mol} / \mathrm{L}$ in the HUA group, and $296.7( \pm 78.2) \mu \mathrm{mol} / \mathrm{L}$ and $312 \mu \mathrm{mol} / \mathrm{L}$ in the non-HUA group, respectively. The univariate analysis revealed that HUA was correlated with blood glucose level, lipid profile, and other metabolic parameters. With the increasing quartiles of fasting glucose, TG, and LDL-C levels, and BMIs from Q1 to Q2 to Q3 to Q4, the risk of HUA also increased. The multivariate logistic regression analysis revealed that after adjustment for the clinical and metabolic factors (model 2), including age, sex, marital status, eGFR, new-onset hypertension, antihypertensive drugs and aspirin use, higher levels of fasting glucose, TG, LDL-C and BMI were still associated with higher risk of HUA (Table 2).

\section{HUA and new-onset bypertension}

The survey showed that the prevalence of HUA in patients with new-onset hypertension (2,785 cases) was up to $45.4 \%$, higher than that in those with an established hypertension (38.1\% , 31,000 cases). With the patients with established hypertension as control, the univariate analysis revealed that the relative risk of HUA increased by $35 \%$ in the patients with new-onset hypertension (OR, 1.35; 95\% CI, 1.251.46). The multivariate logistic regression analysis (model 2) revealed that after adjustment for the clinical and metabolic parameters, new-onset hypertension was still a risk factor for HUA (OR, 2.01; 95\% CI, 1.73-2.33).

In addition, the data showed that the prevalence of HUA in the unmarried hypertensive patients was 49\%, higher than the $39 \%$ in those who were married. The univariate analysis revealed that singlehood increased the risk of HUA (OR, 1.53; 95\% CI, 1.06-2.01). Compared with those who were married, unmarried hypertensive patients had a $58 \%$ increased risk of HUA (OR, 1.58; 95\% CI, 1.10-2.27) in the multivariate logistic regression analysis (model 1), but the risk of HUA was not significant after the inclusion of metabolic parameters (OR, 1.27; 95\% CI, 0.93-1.27; model 2). Despite that the use of aspirin was associated with the increased risk of HUA in model 1, it was not significant as the metabolic parameters were included in model 2 . Female sex, age of $\geq 65$ years, and lower eGFR were independently associated with higher risk of HUA, while use of losartan, valsartan, and nifedipine were associated with lower risk of HUA, with similar results between models 1 and 2 (Table 2).

\section{Discussion}

UA is the ultimate catabolite of purine metabolism in humans and higher primates. HUA is related to the 
occurrence and development of hypertension. Previous studies found that $47 \%$ of the hypertensive population had HUA (11). Epidemiological studies have confirmed that HUA has a strong correlation with the occurrence of hypertension. Pooled analysis revealed that for every $1 \mathrm{mg} / \mathrm{dL}$ increase in serum UA, the risk of hypertension increased by $13 \%$, and this effect was more pronounced in women and young adults (12). An experimental study showed that UA increases $\mathrm{BP}$ at the initial stage, in a UAdependent manner, and that $\mathrm{BP}$ could be reduced with UAlowering medications; however, prolonged HUA results in irreversible sodium-sensitive hypertension that becomes UA independent $(13,14)$. The early stage (UA causes rapid increase in BP) mainly involves an increase in renin activity and a reduction in circulating nitrates, which results in vasoconstriction that can be reversed by administration of UA-lowering agents or RAS inhibitors $(15,16)$. The late and long-term mechanism of high BP may be associated with renal interstitial vascular structural changes. Studies have revealed that UA enters vascular smooth muscle cells (VSMCs) via UA anion transporter-1 channels, which leads to the activation of kinases, nuclear transcription factors, cyclooxygenase 2, platelet-derived growth factor and inflammatory protein (e.g., C-reactive protein and monocyte chemoattractant protein 1), which eventually results in VSMCs proliferation, shifted pressure natriuresis and sodium-sensitive hypertension (17-19). Other studies have found that HUA is associated with increased CVD risk (3-5). Recent genetic evidences based on conventional and novel Mendelian randomization approaches suggest a modest, if any, causal effect of plasma UA concentration on the development of CVDs (20,21). Despite these evidences, the association between serum UA and CVDs is currently considered to be not always independent of traditional risk factors or diseases, such as hypertension, diabetes, or chronic kidney diseases (12).

A previous study demonstrated that the prevalence of HUA in hypertensive patients in a rural area (Xinyang) in China was approximately $14 \%$ (7). In this survey, HUA was found in approximately two-fifth of hypertensive patients. The major difference might reflect the transition of the lifestyle and dietary structure of the residents in China. Increasing intake of the high-calorie diet with meat, fruit juice, or other fructose-rich drinks and foods is related to the development of HUA.

The occurrence of HUA in hypertensive patients is affected by many factors. Aging was an independent predictor of HUA, and the risk of HUA increased in the hypertensive patients aged $\geq 65$ years in the survey. Compared with the males, the female hypertensive patients had a significant increase in the risk of HUA, which may be due to the elevated serum UA level in the postmenopausal period (22). Excretion of urate decreased with impaired renal filtration, as the survey demonstrated that lower eGFR was associated independently with higher risk of HUA. The study also found that the prevalence of HUA in the residents of Western China was significantly higher than that in the residents of the Eastern region, which may be related to the dietary structure and habits of the residents in these regions. The Western residents had the tendency to have a higher intake of purine-rich meat, while the Eastern residents had a relatively lighter diet. In addition, longer hypertension duration, lower educational level (high school or lower), singlehood, metabolic disorders (e.g., impaired glucose tolerance/diabetes, dyslipidemia, and obesity), and aspirin use are potential risk factors for HUA in separate models adjusted for demographic and clinical confounders. These factors identified in the survey are routinely collected in clinical practice and may be readily incorporated into risk prediction. In this study, the univariate and multivariate regression analyses both revealed that singlehood was a predictive factor for HUA, which may be explained by the fact that most of the unmarried patients were younger, had intemperate diet, or had other risk factors. However, the result should be interpreted cautiously, as the proportion of unmarried people in the survey was $<1 \%$ (only 202 cases), and bias could not be excluded. In addition, the present study showed that the prevalence of HUA in the patients with new-onset hypertension was higher than in those with established hypertension. However, owing to the small number of patients in this group (2,570 cases), this finding should also be interpreted with caution.

Moreover, for hypertensive patients receiving antihypertensive medications, the class of drugs can be associated with HUA occurrence. A study of data from the UK general practice database showed that different antihypertensive drugs had different effects on HUAderived gout. CCBs and losartan had an attenuating effect on gout, whereas diuretics, $\beta$ blockers, ACEIs and other non-losartan ARBs increased the risk of gout. Further analysis indicated that the attenuating effect of CCBs on gout was time dependent; that is, CCBs slightly increased the risk of gout in the first year, but decreased it gradually thereafter on a yearly basis. The effect of losartan was also time dependent, showing a continuously decreasing trend (23). A community study (ARIC) in the United States 
suggested that non-diuretic antihypertensive medications significantly reduced the incidence of gout in hypertensive patients, as compared with untreated patients, with an adjusted hazard ratio (HR) of 0.64 (95\% CI, 0.49-0.86). Compared with non-diuretics, diuretics (either thiazide, or loop diuretics) significantly increased the risk of gout (HR, 1.48; 95\% CI, 1.11-1.98). Compared with non-thiazide diuretics, thiazides increased risk of gout (HR, 1.44; $95 \%$ CI, 1.00-2.01). Use of loop diuretics also demonstrated a significant increase in the risk of gout, as compared with the use of non-loop diuretics (HR, 2.31; 95\% CI, 1.36-3.91) (8). A study on a hypertensive population in Taiwan found that use of diuretics and $\beta$ blockers could predict HUA, while the use of ACEIs, CCBs and ARBs had little relationship with the occurrence of HUA (24). A study in Japan showed that administration of diuretics, $\beta$-blockers, and $\alpha$-blockers increased serum UA levels in hypertensive patients, while use of CCBs, ACEIs, ARBs, including losartan did not increase UA levels (25). In our investigation, we found that the use of antihypertensive agents, including ARBs, ACEIs, CCBs or $\beta$ blockers, was associated with a lower risk of HUA compared with no treatment for hypertension. Further analysis revealed that losartan, valsartan, nifedipine and $\beta$-blockers were protective factors for HUA, with lower risk of HUA in the patients who were taking these medications. Other antihypertensive drugs including amlodipine and diuretics did not significantly increase the risk of HUA. Previous literature showed that losartan, an $\mathrm{ARB}$, could promote UA excretion, thereby reducing UA levels $(9,10)$. Except for losartan, other ARBs did not show definite UA-lowering effects (26). Controlled-release nifedipine was also found to be effective for reducing serum UA levels in patients with coronary heart disease in the ACTION trial (27), and the uricosuric effect was confirmed in the urate under-excretion mice model (28). The findings of this study are generally in consistent with the results of previous studies. However, the protective effect of $\beta$-blockers for HUA should be interpreted with caution. First, only few patients in this study took $\beta$ blockers, accounting for only $2.7 \%$ of the total population; thus, the findings may be biased. Second, metoprolol and atenolol have been reported to increase UA levels $(29,30)$, while celiprolol, a vasodilating $\beta$-blocker, has a tendency to reduce UA levels slightly (31). Moreover, this investigation found that diuretics showed a tendency to increase the risk of HUA, but it was not statistically significant, which may be due to the low proportion and quantity of patients who were taking diuretics, and the low statistical power.

Our study has several limitations. Owing to its crosssectional design, we could not analyze the effects of the dynamic changes of the metabolic parameters such as glucose or lipid profiles, as well as the antihypertensive agents on HUA. To avoid affecting the result of the HUA prevalence, we excluded patients with hypertension comorbid with gout or those who had been taking urate-lowering therapeutic medications, including allopurinol, febuxostat or benzbromarone, etc. from the survey. While losartan, with the uricosuric potential, might still have a modest impact on the result. As we exclude the patients who had been taking losartan, and recalculate the data, the prevalence of HUA will be $1 \%$ higher than the $38.7 \%$ in the overall population. Although we included demographic or clinical parameters in the different models in the logistic regression analysis, some factors with predictive potential might have been neglected. As aforementioned, some bias could exist owing to the small proportion or number of the patients under the different categories. In addition, as this study included only Chinese patients with hypertension, the results of the survey should not be extrapolated to other ethnicities.

\section{Conclusions}

This large-sample population survey showed that the prevalence of HUA in Chinese hypertensive patients was $38.7 \%$. Female sex, aging ( $\geq 65$ years) and low eGFR were independent risk factors for HUA. The prevalence of HUA was lower among the patients who were taking losartan, valsartan and nifedipine. Western region residents, newonset hypertension, longer hypertension duration, aspirin use, and higher FBG, TG, and LDL-C levels and BMI might be associated with increased risk of HUA.

\section{Acknowledgments}

The authors acknowledge all the participants and physicians involved in the survey. The authors thank Editage (www. editage.cn) for English language editing during the manuscript revision.

\section{Footnote}

Reporting Checklist: The authors have completed the STROBE reporting checklist. Available at http://dx.doi. org/10.21037/atm-20-3458 
Data Sharing Statement: Available at http://dx.doi. org/10.21037/atm-20-3458

Conflicts of Interests: All authors have completed the ICMJE uniform disclosure form (available at http://dx.doi. org/10.21037/atm-20-3458). The authors report that Salibruis Pharmceutical Co. (Shenzhen) sponsored the survey, but the data and opinions in text were not influenced by the sponsor. The authors have no other conflicts of interest to declare.

Ethical Statement: The authors are accountable for all aspects of the work in ensuring that questions related to the accuracy or integrity of any part of the work are appropriately investigated and resolved. This study was in accordance with the Declaration of Helsinki (as revised in 2013). The protocol was approved by the central institutional review board of Peking University First Hospital (No. 2018-102). Written informed consent was obtained from all participants.

Open Access Statement: This is an Open Access article distributed in accordance with the Creative Commons Attribution-NonCommercial-NoDerivs 4.0 International License (CC BY-NC-ND 4.0), which permits the noncommercial replication and distribution of the article with the strict proviso that no changes or edits are made and the original work is properly cited (including links to both the formal publication through the relevant DOI and the license). See: https://creativecommons.org/licenses/by-nc-nd/4.0/.

\section{References}

1. Han M, Park HC, Kim H, et al. Hyperuricemia and deterioration of renal function in autosomal dominant polycystic kidney disease. BMC Nephrol 2014;15:63.

2. Feig DI. Serum uric acid and the risk of hypertension and chronic kidney disease. Curr Opin Rheumatol 2014;26:176-85.

3. Virdis A, Masi S, Casiglia E, et al. Identification of the uric acid thresholds predicting an increased total and cardiovascular mortality over 20 years. Hypertension 2020;75:302-8.

4. Verdecchia P, Schillaci G, Reboldi G, et al. Relation between serum uric acid and risk of cardiovascular disease in essential hypertension. The PIUMA study. Hypertension 2000;36:1072-8.

5. Cicero AF, Salvi P, D'Addato S, et al. Association between serum uric acid, hypertension, vascular stiffness and subclinical atherosclerosis: Data from the Brisighella Heart Study. J Hypertens 2014;32:57-64.

6. Ofori SN, Odia OJ. Serum uric acid and target organ damage in essential hypertension. Vasc Health Risk Manag 2014;10:253-61.

7. Fan XH, Sun K, Wang YB, et al. Prevalence and associated risk factors of hyperuricemia in rural hypertensive patients. Zhonghua Yi Xue Za Zhi 2009;89:2667-70.

8. McAdams DeMarco MA, Maynard JW, Baer AN, et al. Diuretic use, increased serum urate levels and risk of incident gout in a population-based study of adults with hypertension. Arthritis Rheum 2012;64:121-9.

9. Ohshiro K, Sakima A, Nakada S, et al. Beneficial effect of switching from a combination of angiotensin II receptor blockers other than losartan and thiazides to a fixed dose of losartan/hydrochlorothiazide on uric acid metabolism in hypertensive patients. Clin Exp Hypertens 2011;33:565-70.

10. Hamada T, Ichida K, Hosoyamada M, et al. Uricosuric action of losartan via the inhibition of urate transporter 1 (URAT 1) in hypertensive patients. Am J Hypertens 2008;21:1157-62.

11. Cannon PJ, Stason WB, Demartini FE, et al. Hyperuricemia in primary and renal hypertension. $\mathrm{N}$ Engl J Med 1966;275:457-64.

12. Grayson PC, Kim SY, LaValley M, et al. Hyperuricemia and incident hypertension: a systematic review and metaanalysis. Arthritis Care Res (Hoboken) 2011;63:102-10.

13. Mazzali M, Hughes J, Kim YG, et al. Elevated uric acid increases blood pressure in the rat by a novel crystalindependent mechanism. Hypertension 2001;38:1101-6.

14. Mazzali M, Kanellis J, Han L, et al. Hyperuricemia induces a primary renal arteriolopathy in rats by a blood pressure independent mechanism. Am J Physiol Renal Physiol 2002;282:F991-7.

15. Sánchez-Lozada LG, Tapia E, López-Molina R, et al. Effects of acute and chronic L-arginine treatment in experimental hyperuricemia. Am J Physiol Renal Physiol 2007;292:F1238-44.

16. Sautin YY, Nakagawa T, Zharikov S, et al. Adverse effects of the classic antioxidant uric acid in adipocytes: NADPH oxidasemediated oxidative/nitrosative stress. Am J Physiol Cell Physiol 2007;293:C584-96.

17. Kanellis J, Watanabe S, Li JH, et al. Uric acid stimulates monocyte chemoattractant protein-1 production in vascular smooth muscle cells via mitogen-activated protein kinase and cyclooxygenase-2. Hypertension 
2003;41:1287-93.

18. Price KL, Sautin YY, Long DA, et al. Human vascular smooth muscle cells express a urate transporter. J Am Soc Nephrol 2006;17:1791-5.

19. Feig DI. Hyperuricemia and hypertension. Adv Chronic Kidney Dis 2012;19:377-85.

20. Kleber ME, Delgado G, Grammer TB, et al. Uric acid and cardiovascular events: A Mendelian randomization study. J Am Soc Nephrol 2015;26:2831-8.

21. White J, Sofat R, Hemani G, et al. Plasma urate concentration and risk of coronary heart disease: A Mendelian randomization analysis. Lancet Diabetes Endocrinol 2016;4:327-36.

22. Hak AE, Choi HK. Menopause, postmenopausal hormone use and serum uric acid levels in US women - The Third National Health and Nutrition Examination Survey. Arthritis Res Ther 2008;10:R116.

23. Choi HK, Soriano LC, Zhang Y, et al. Antihypertensive drugs and risk of incident gout among patients with hypertension: population based case-control study. BMJ 2012;344:d8190.

24. Lin CS, Lee WL, Hung YJ, et al. Prevalence of hyperuricemia and its association with antihypertensive treatment in hypertensive patients in Taiwan. Int J Cardiol 2012;156:41-6.

25. Ueno S, Hamada T, Taniguchi S, et al. Effect of

Cite this article as: Liu J, Chen L, Yuan H, Huang K, Li G, Sun N, Huo Y; on behalf of the SUCCESS investigation group. Survey on uric acid in Chinese subjects with essential hypertension (SUCCESS): a nationwide cross-sectional study. Ann Transl Med 2021;9(1):27. doi: 10.21037/atm-20-3458 antihypertensive drugs on uric acid metabolism in patients with hypertension: cross-sectional cohort study. Drug Res (Stuttg) 2016;66:628-32.

26. Wolff ML, Cruz JL, Vanderman AJ, et al. The effect of angiotensin II receptor blockers on hyperuricemia. Ther Adv Chronic Dis 2015;6:339-46.

27. Ruilope LM, Kirwan BA, de Brouwer S, et al. Uric acid and other renal function parameters in patients with stable angina pectoris participating in the ACTION Trial: impact of nifedipine GITS and relation to outcome. J Hypertens 2007;25:1711-8.

28. Hori T, Ouchi M, Otani N, et al. The uricosuric effects of dihydropyridine calcium channel blockers in vivo using urate under-excretion animal models. J Pharmacol Sci 2018;136:196-202.

29. Juraschek SP, Appel LJ, Miller ER 3rd. Metoprolol increases uric acid and risk of gout in African Americans with chronic kidney disease attributed to hypertension. Am J Hypertens 2017;30:871-5.

30. Andersen GS. Atenolol versus bendroflumethiazide in middle-aged and elderly hypertensives. Acta Med Scand 1985;218:165-72.

31. Mizuta E, Utami SB, Ohtahara A, et al. A vasodilating $\beta 1$ blocker celiprolol inhibits muscular release of uric acid precursor in patients with essential hypertension. Horm Metab Res 2013;45:69-73. 